\title{
Carbon Nanoparticle-based Fluorescent Bioimaging Probes
}

SUBJECT AREAS: NANOPARTICLES

BIOSENSORS

DIAGNOSTIC MARKERS

COLIOIDS

Received

11 December 2012

Accepted

4 March 2013

Published

18 March 2013

Correspondence and requests for materials should be addressed to N.R.J. (camnri@iacs. res.in)
Susanta Kumar Bhunia', Arindam Saha', Amit Ranjan Maity', Sekhar C. Ray² \& Nikhil R. Jana'

${ }^{1}$ Centre for Advanced Materials, Indian Association for the Cultivation of Science, Kolkata-700032, India, ${ }^{2}$ School of Physics, University of the Witwatersrand, Private Bag 3, Wits-2050, Johannesburg, South Africa.

Fluorescent nanoparticle-based imaging probes have advanced current labelling technology and are expected to generate new medical diagnostic tools based on their superior brightness and photostability compared with conventional molecular probes. Although significant progress has been made in fluorescent semiconductor nanocrystal-based biological labelling and imaging, the presence of heavy metals and the toxicity issues associated with heavy metals have severely limited the application potential of these nanocrystals. Here, we report a fluorescent carbon nanoparticle-based, alternative, nontoxic imaging probe that is suitable for biological staining and diagnostics. We have developed a chemical method to synthesise highly fluorescent carbon nanoparticles $1-10 \mathrm{~nm}$ in size; these particles exhibit size-dependent, tunable visible emission. These carbon nanoparticles have been transformed into various functionalised nanoprobes with hydrodynamic diameters of 5-15 $\mathrm{nm}$ and have been used as cell imaging probes.

luorescent carbon nanoparticles (FCN) with tunable emission are considered to be next generation green nanomaterials ${ }^{1-8}$ and are promising alternatives to fluorescent semiconductor nanocrystals, which are composed of toxic heavy metals such as cadmium ${ }^{9,10}$. FCNs have been demonstrated to be potential optical detection probes $^{8}$, bioimaging probes ${ }^{3}$, light emitting diode materials ${ }^{7}$ and efficient visible light-active photocatalysts ${ }^{6}$. The potential of FCNs in biomedical applications is enormous because the major component is the nontoxic element carbon. However, this potential is relatively unexplored compared to that of other carbon-based materials, such as fullerenes ${ }^{11}$, carbon nanotubes ${ }^{12}$ and graphene ${ }^{13}$ largely because high quality FCNs and probes of FCN have been unavailable. FCNs have been synthesised through physical methods, such as high energy radiation-based creation of point defects in diamond ${ }^{14,15}$ and laser ablation of graphite ${ }^{16}$, or through chemical methods, such as oxidation of candle soot ${ }^{17,18}$, carbonisation of carbohydrates ${ }^{19-23}$, thermal decomposition of small molecules ${ }^{24-28}$, pyrolysis of polymers ${ }^{29}$, microwave-based pyrolysis ${ }^{30-32}, \mathrm{P}_{2} \mathrm{O}_{5}$-based room temperature dehydration of small molecules ${ }^{33}$, electrochemical methods ${ }^{34,35}$ and chemical breakdown of carbon fiber ${ }^{36}$, graphene $^{37}$ or graphite ${ }^{38}$. However, there are four distinct limitations of the currently available FCN. (Supplementary Table S1) First, most of the synthetic methods produce weakly fluorescent FCNs with a quantum yield of less than $1 \%$. Second, no methods are currently available for the large scale synthesis of high-quality FCNs. Although some methods report milligram-scale FCNs with quantum yields of 5 to $60 \%$, these methods require a sophisticated high energy radiation-based synthesis followed by surface functionalisation and size separation ${ }^{2}$. Third, although many methods report blue- and green-emitting FCNs, only a few methods report yellow- and red-emitting FCNs. In addition, yellow- and red-emitting FCNs are generally mixed with blue- and green-emitting FCNs and must be isolated through specialised size separation methods $s^{2,17,34,38}$. Fourth, although the functionalisation of nanoprobes is essential to the enhancement of the labelling specificity, such strategies are poorly developed for $\mathrm{FCNs}^{3,15,18,19,25,33,36}$, and in most cases, bare FCNs have been used for bioimaging and other applications ${ }^{15,18,19,33,36}$.

We report chemically synthesised, highly fluorescent FCNs suitable for biological labelling and imaging applications. These FCNs have tunable emissions (such as blue, green, yellow and red) and quantum yields between 6 and 30\%, have been synthesised on the milligram to gram scale and have been transformed into various functionalised nanoprobes for different biological labelling and imaging applications. The well-known carbohydrate carbonisation method has been exploited for the FCN synthesis; this method usually produces non-fluorescent carbon particles greater than $10 \mathrm{~nm}$ in $\operatorname{size}^{39}$ or weakly fluorescent, blue- or green-emitting carbon particles $^{20}$. Here, we have extensively varied the carbonisation conditions to finely tune nucleation and growth; thus, carbon nanoparticles less than $10 \mathrm{~nm}$ in size can be prepared with a narrow size distribution. (Figure 1) Two different approaches have been used to control the nucleation and growth. In the first approach, different carbohydrate molecules that dehydrate at different temperatures were used as carbon sources. In the second approach, the dehydrating agent and the reaction temperature were varied to control the nucleation and growth 


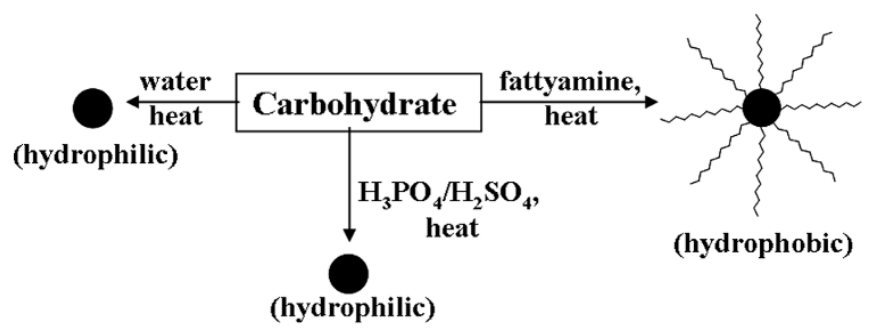

Figure $1 \mid$ Synthesis of fluorescent carbon nanoparticle. Different controlled carbonisation approach of carbohydrate in producing fluorescent carbon nanoparticle (FCN).

kinetics. The presented synthetic method restricts the particle size to smaller than $10 \mathrm{~nm}$ and provides an appropriate chemical composition for the FCNs for tunable emission with a high fluorescence quantum yield. (Table 1, Figure 2 and Supplementary Figures S1 and S2) These FCNs have been transformed into functionalised nanoprobes without significant loss of their fluorescence properties through conventional bioconjugation chemistries. Functionalised FCN-based nanoprobes have tunable and stable visible emission similar to that of semiconductor nanoparticle-based nanoprobes but with the advantage of small hydrodynamic size and low toxicity. The small size of the nanoprobes affords easier subcellular targeting, whereas the low toxicity allows wider applications for the nanoprobes.

\section{Results}

A variety of carbohydrates and different nucleation and growth conditions have been used to obtain FCNs with tunable emission and with high quantum yield. The tested molecules include glucose, glucosamine, sucrose, dextran of different molecular weights, cellulose and ascorbic acid. Carbonisation has been performed in different solvents (e.g., octadecene, octadecene-fatty amine, octadecene-fatty acid, ethylene glycol and water) with different dehydrating agents (e.g., $\mathrm{H}_{3} \mathrm{PO}_{4}, \mathrm{H}_{2} \mathrm{SO}_{4}$ ) and at various temperatures from 80 to $300^{\circ} \mathrm{C}$. Although higher temperatures $\left(150-300^{\circ} \mathrm{C}\right)$ increase the rate of carbonisation, the promotion of rapid nucleation over growth restricts the FCNs to $<4 \mathrm{~nm}$ in size, and the resultant emission colour of the FCNs is limited to blue and green. $\mathrm{H}_{3} \mathrm{PO}_{4}$ - and $\mathrm{H}_{2} \mathrm{SO}_{4}$-based carbonisations at lower temperatures $\left(80-120^{\circ} \mathrm{C}\right)$ afford particle sizes between 1 and $10 \mathrm{~nm}$ and produce blue-, green-, yellow- and redemitting FCNs. However, the reaction conditions must be finely adjusted to minimise particle aggregation.

In a typical procedure, the carbohydrate is dissolved in a solvent or is mixed with dehydrating agents, and carbonisation is performed at the appropriate temperature. In one approach, carbonisation of the carbohydrate is performed in octadecene solvent in the presence of a long chain fatty amine. These synthetic conditions are similar to the well-established synthetic conditions for metal/metal oxide/semiconductor nanoparticles ${ }^{40}$ and produce hydrophobic FCNs with a fatty amine cap. This approach is most suitable for the synthesis of blue- and green-emitting FCNs. Blue- and green-emitting FCNs can also be prepared in boiling aqueous carbohydrate solutions, but this approach is very selective to the few carbohydrates with lower carbonisation temperatures. Sulphuric acid-based carbonisation at 50$100^{\circ} \mathrm{C}$ mostly produces blue- and green-emitting FCNs. The best condition for yellow- and red-emitting FCNs is concentrated phosphoric acid-based carbonisation of carbohydrates at $80-90^{\circ} \mathrm{C}$.

Figure 2 shows the optical properties of four types of FCNs with different emission colours. Although all of the samples show excitation-dependent emission spectra, each sample has its most intense emission for a certain excitation. (Supplementary Figure S1) The four FCN samples are classified as $\mathrm{FCN}_{\text {blue }}, \mathrm{FCN}_{\text {green }}, \mathrm{FCN}_{\text {yellow }}$ and $\mathrm{FCN}_{\text {red }}$ based on their most intense emission at $440 \mathrm{~nm}$ (excitation at $370 \mathrm{~nm}$ ), $500 \mathrm{~nm}$ (excitation at $400 \mathrm{~nm}$ ), $560 \mathrm{~nm}$ (excitation at $425 \mathrm{~nm}$ ) and $600 \mathrm{~nm}$ (excitation at $385 \mathrm{~nm}$ ), respectively. The fluorescence quantum yields (QY) for these samples range between 6 and $30 \%$ and are measured in reference to fluorescein or quinine sulfate ${ }^{41}$. A comparative study shows that the band edges for the FCN absorption red shift from $400 \mathrm{~nm}$ to $600 \mathrm{~nm}$ as the excitation maxima red shift. The particle sizes of the FCNs were determined with Transmission electron microscopy (TEM) and size exclusion chromatography (SEC). $\mathrm{FCN}_{\text {red }}, \mathrm{FCN}_{\text {yellow }}$ and hydrophobic $\mathrm{FCN}_{\text {green }}$ are seen with TEM, but FCN $\mathrm{Flue}_{\text {blu }}$ and hydrophilic $\mathrm{FCN}_{\text {green }}$ are too small and the contrast is too poor to be seen with TEM. The particle sizes determined by TEM are 2-4 nm, 1-4 nm and 4-10 nm for $\mathrm{FCN}_{\text {green }}$ (hydrophobic), $\mathrm{FCN}_{\text {yellow }}$ and $\mathrm{FCN}_{\text {red }}$, respectively. (Figure 3 and Supplementary Figure S2) SEC study of hydrophilic FCN $\mathrm{F}_{\text {blue }}$ and $\mathrm{FCN}_{\text {green }}$ shows that they have molecular weights in the range of $0.4-2.2 \mathrm{kDa}$ and $2.5-14 \mathrm{kDa}$, respectively. The molar extinction coefficients for all the FCNs have been determined similarly to those of quantum dots ${ }^{42}$ and gold nanoparticles ${ }^{43}$, assuming nanoparticle as single entity.

The compositions of the FCNs have been characterised by different methods, such as elemental analysis, XPS, Fourier transform infrared spectroscopy (FTIR) and proton NMR spectroscopy. (Supplementary Figures S3-S8) Elemental analysis shows the presence of elemental carbon as the major component (50-75\%) and other minor components, such as hydrogen, nitrogen, oxygen and phosphorous. The composition varies based on the nature of the precursor carbohydrate and the presence of surface-adsorbed molecules. (Table 1) XPS analysis further confirms the presence of trace nitrogen and phosphorous in some of the FCNs; these elements cannot be detected by conventional CHN analysis. Proton NMR and FTIR studies show the signatures of surface functional groups such as $\mathrm{OH}, \mathrm{CH}, \mathrm{COOH}, \mathrm{NH}$ and $\mathrm{NH}_{2}$. The ratio of $\mathrm{sp}^{2}$ to $\mathrm{sp}^{3}$ carbons is determined by XPS and shows that there is a significant percentage of $\mathrm{sp}^{3}$ carbon with the $\mathrm{sp}^{2}$ carbon. Raman studies show the presence of the $\mathrm{D}$ and $\mathrm{G}$ band, and the large intensity ratio of the $\mathrm{D}$ to $\mathrm{G}$ band indicates the existence of defects in the FCNs. (Figure 4) The presence of defect sites is also confirmed by electron paramagnetic resonance (EPR) signals that correspond to free electrons, which are observed at room temperature for all the types of FCNs. (Figure 4) XRD shows broad peaks centred at $2 \theta \sim 20^{\circ}$, which corresponds to highly amorphous carbon, and shows a weak peak at $2 \theta \sim 40^{\circ}$, which corresponds to the $\mathrm{d}$ (100) plane. Magnetic measurement studies do not show any significant magnetic properties. The lifetime decays of all of the FCNs fit within 2-3 lifetime

Table 1 | Property of different fluorescent carbon nanoparticles (FCN)

\begin{tabular}{|c|c|c|c|c|c|}
\hline $\mathrm{FCN}$ & $\begin{array}{l}\text { Molecular weight/ } \\
\text { Particle Size }\end{array}$ & $\begin{array}{c}\text { Elemental composition } \\
\text { (C:H:N:O) }\end{array}$ & $\begin{array}{l}\text { Emission }_{\text {max }} \\
\text { (excitation) }\end{array}$ & $\begin{array}{l}\text { Molar extinction } \\
\text { coefficient at excitation }\end{array}$ & $\begin{array}{l}\text { Fluorescence } \\
\text { quantum yield }\end{array}$ \\
\hline $\begin{array}{l}\mathrm{FCN} \text { bule } \\
\mathrm{FCN}_{\text {green }} \\
\mathrm{FCN}_{\text {yellow }} \\
\mathrm{FCN}_{\text {red }}\end{array}$ & $\begin{array}{l}400-2200 \mathrm{Da} \\
2500-14000 \mathrm{Da}, 2-4 \mathrm{~nm} \\
\quad 1-4 \mathrm{~nm} \\
\sim 4-10 \mathrm{~nm}\end{array}$ & $\begin{array}{l}65: 6: 8: 21 \\
75: 10: 5: 10 \\
50: 15: 2: 33 \\
70: 5: 1: 24\end{array}$ & $\begin{array}{l}440 \mathrm{~nm}(370 \mathrm{~nm}) \\
500 \mathrm{~nm}(400 \mathrm{~nm}) \\
560 \mathrm{~nm}(425 \mathrm{~nm}) \\
600 \mathrm{~nm}(385 \mathrm{~nm})\end{array}$ & $\begin{array}{l}2 \times 10^{3} \\
5 \times 10^{4} \\
4 \times 10^{3} \\
7 \times 10^{5}\end{array}$ & $\begin{array}{r}6-30 \% \\
14 \% \\
12 \% \\
7 \%\end{array}$ \\
\hline
\end{tabular}



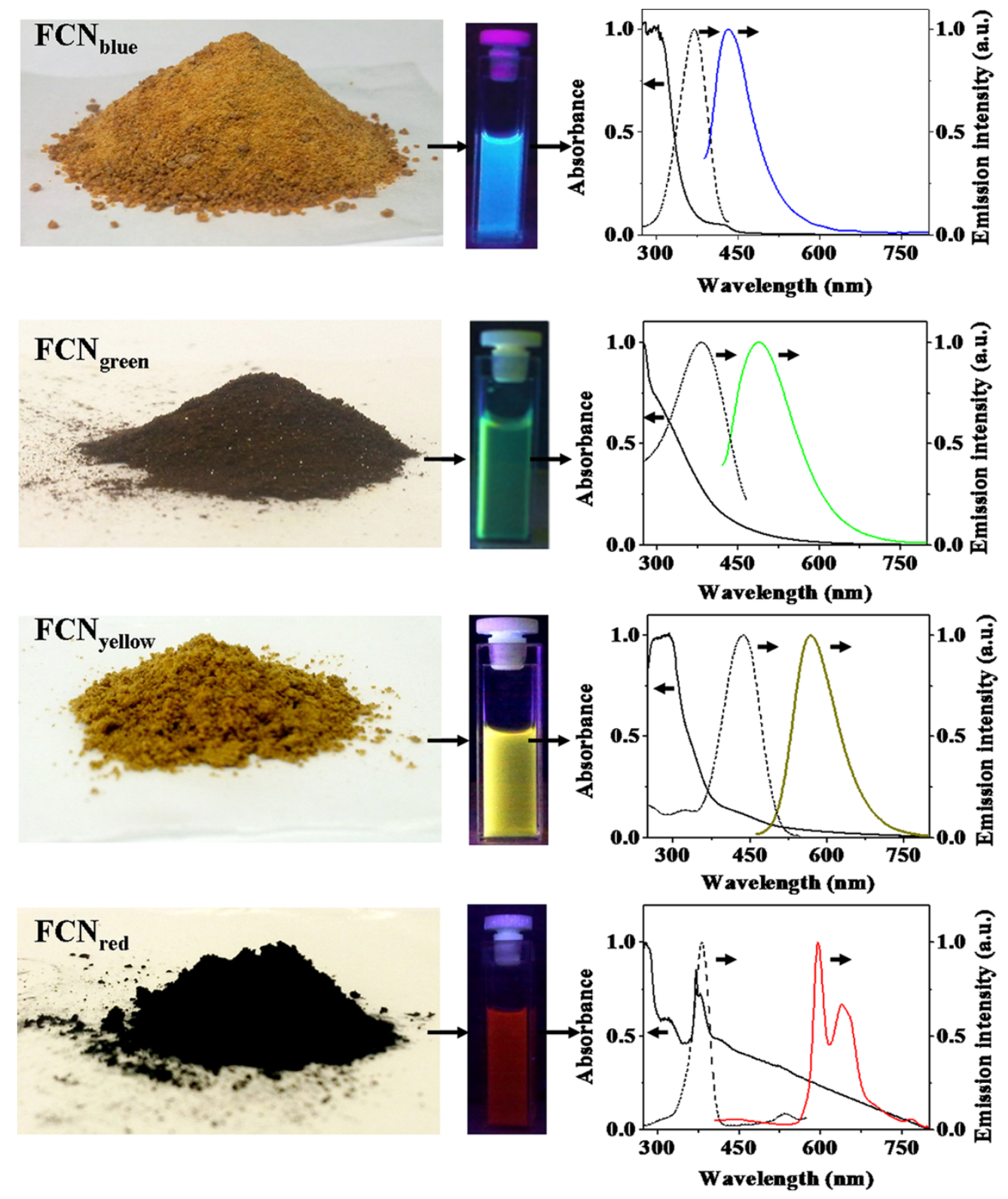

Figure $2 \mid$ Optical property of fluorescent carbon nanoparticle. Digital image of gram scale solid FCN samples, digital images of their solutions under appropriate excitations and their absorption (-), excitation (.....) and emission (color lines) spectra. Emission spectra have been measured by exciting at $370 \mathrm{~nm}$ for $\mathrm{FCN}_{\text {blue }}$, by exciting at $400 \mathrm{~nm}$ for $\mathrm{FCN}_{\text {green }}$, by exciting at $425 \mathrm{~nm}$ for $\mathrm{FCN}_{\text {yellow }}$ and by exciting at $385 \mathrm{~nm}$ for FCN $\mathrm{Ned}_{\text {. }}$ All excitation spectra are recorded in respective emission maxima.

components on the $0.1-10$ nanosecond time scale; this result suggests that multiple radiative species are present in each sample. (Supplementary Figure S9).

To extend the application potential of FCNs as fluorescent labels, we have investigated the fluorescence stability of FCNs under different conditions. (Supplementary Figure S10-S12) The fluorescence of FCNs, either in solution or as a solid film, remains stable under continuous exposure to light, suggesting that the FCNs do not photobleach significantly under exposure to light and can be used as a stable imaging probe. The fluorescence of most of the FCNs is stable in solutions at different $\mathrm{pH}$ values. However, the fluorescence of $\mathrm{FCN}_{\text {red }}$ is only stable in strongly acidic conditions and rapidly transforms to $\mathrm{FCN}_{\text {green }}$ at neutral or basic $\mathrm{pH}$. Thus, we have further processed $\mathrm{FCN}_{\text {red }}$ to stabilise its fluorescence. We found that functionalisation of $\mathrm{FCN}_{\text {red }}$ with polyethylene glycol stabilises the red fluorescence at physiological pH. (Supplementary Figure S10).
Because various functionalised FCNs are required for different bio-labelling applications, it is necessary to test if the as-synthesised hydrophobic and hydrophilic FCNs can be converted into functionalised FCNs that retain their fluorescence. We have successfully transformed all of the FCNs into different functionalised FCNs through conventional coating and conjugation chemistry and have shown their potentials as biological labels. (Figure 5 and Supplementary Figure S13-S19) A hydrophobic FCN can be transformed into a water-soluble FCN through a well-established amphiphilic polymer coating approach ${ }^{44,45}$. In this process, the long hydrocarbon chains of polymers are anchored to the fatty amine shell of the FCN, and the hydrophilic anhydride groups of the polymer react with a controlled amount of PEG-diamine. As a result, the hydrophobic FCN is transformed into a polymer-coated, hydrophilic FCN with terminal primary amine and carboxylate functional groups. The polymer-coated FCN is then functionalised with different affinity 
a)
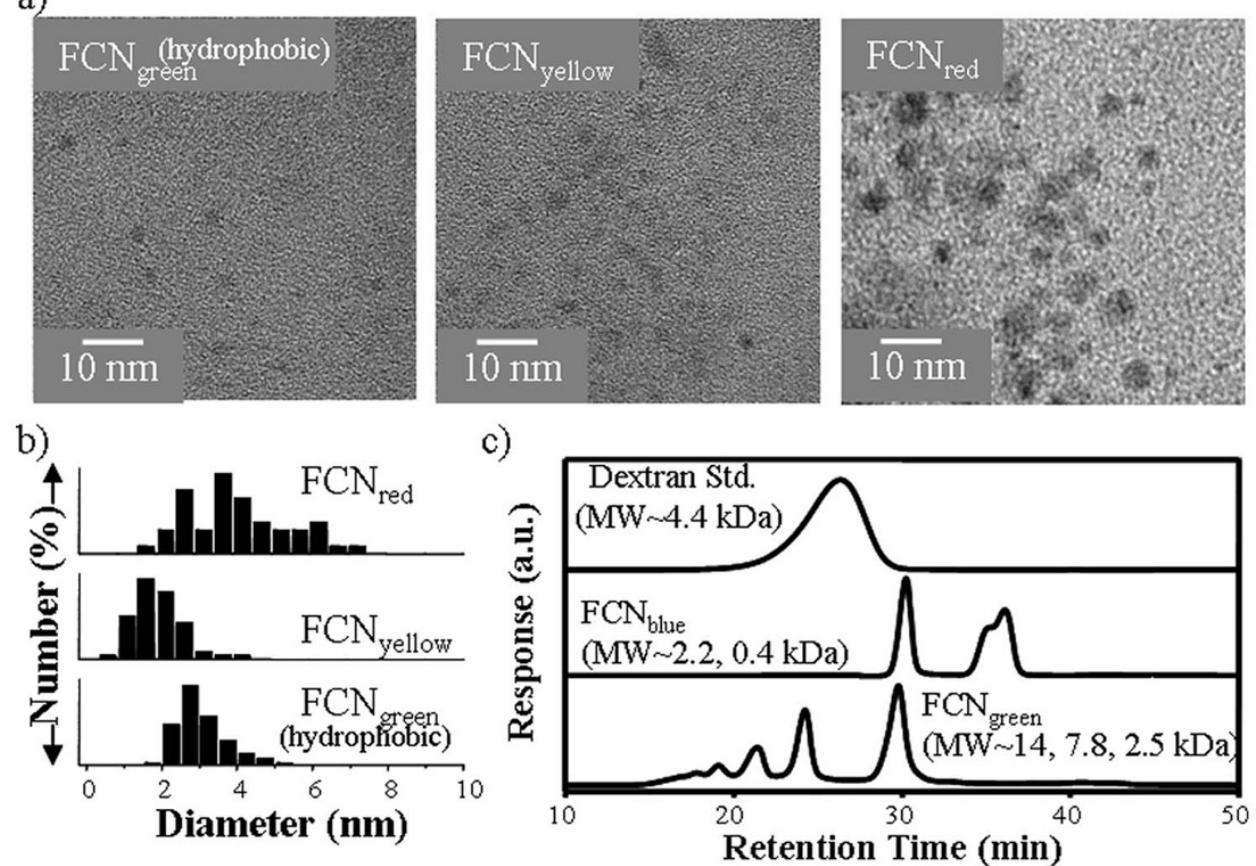

Figure 3 Size characterization of fluorescent carbon nanoparticle. (a) TEM images of hydrophobic $\mathrm{FCN}_{\text {green }}\left(\mathrm{h}_{\mathrm{d} d r o p h o b i c}\right)$, FCN $\mathrm{Fellow}_{\text {and }}$ FCN $\mathrm{Fed}_{\text {red }}$ (b) Size distribution histogram of hydrophobic $\mathrm{FCN}_{\text {green }}$, $\mathrm{FCN}_{\text {red }}$ and $\mathrm{FCN}_{\text {yellow }}$ obtained from TEM images and c) GPC based molecular weight determination for FCN which are TEM invisible showing that $\mathrm{FCN}_{\text {blue }}$ has molecular weight between $0.4-2.2 \mathrm{kDa}$ and $\mathrm{FCN}_{\text {green }}$ has molecular weight between 2.5-14 kDa.

molecules through different conjugation chemistry of the primary amine and carboxylate functional groups. The hydrodynamic sizes of the functionalised FCNs are relatively large (typically, 5-15 nm) compared to those of the as-synthesised FCNs because of the coating layer of polymer. (Supplementary Figure S18) In addition, this functionalisation approach is restricted to $\mathrm{FCN}_{\text {blue }}$ and $\mathrm{FCN}_{\text {green }}$ because the other colours of hydrophobic FCNs are difficult to make by this approach.

Hydrophilic FCNs can be directly transformed into functionalised FCNs through the primary amine and carboxylate groups present on the surface of the FCNs. These amine and acid functional groups either arise from the precursor monomers or from the capping ligands. (Supplementary Figure S14) This approach has been used to prepare TAT peptide- and folate-functionalised FCNs through conjugation chemistry ${ }^{45,46}$. We found that this approach produces functionalised FCNs with a small hydrodynamic diameter $(<15 \mathrm{~nm})$ and is applicable to all types of hydrophilic FCNs. Two types of functionalisation studies show that all the as-synthesised FCNs can be successfully transformed into functionalised FCNs that retain their fluorescence and can be used for different biomedical applications.

To prove that the functionalised FCNs are useful as imaging probes we have investigated their performance as fluorescent cell labels. (Figure 5) TAT peptide- or folate-functionalised FCNs are mixed with cell culture medium, and the cells are attached to culture plates. After incubation for several hours, the washed cells are imaged under a fluorescence microscope. We found that cells are labelled within 1-2 hours and that the labelled cells can be imaged with a a)

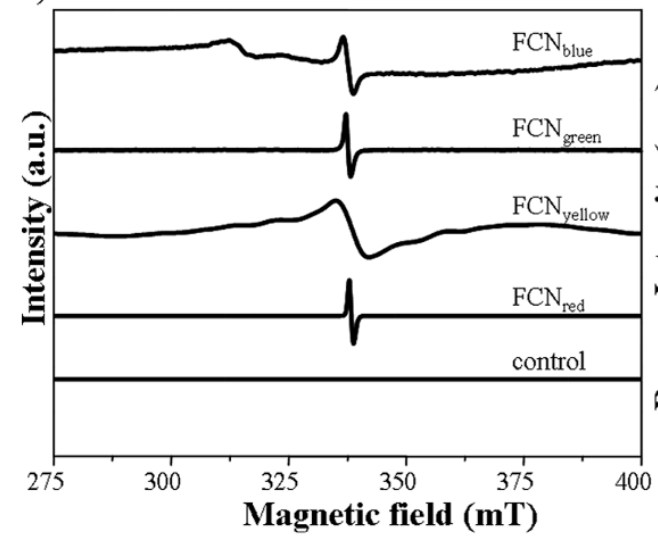

b)

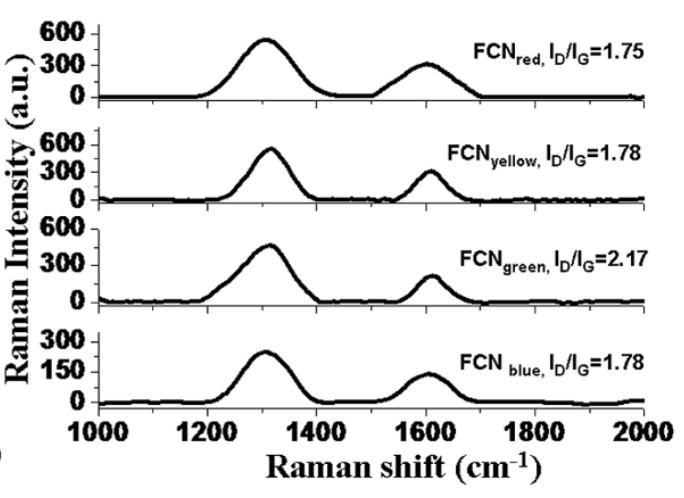

Figure 4 Evidence of defect sites in fluorescent carbon nanoparticle. (a) EPR spectra of FCN measured at $25^{\circ} \mathrm{C}$ in comparison to a control carbon nanoparticles sample having poor fluorescence. (b) Raman spectra of FCNs measured at $785 \mathrm{~nm}$ laser excitation showing prominent D and G bands along with their intensity ratio. 

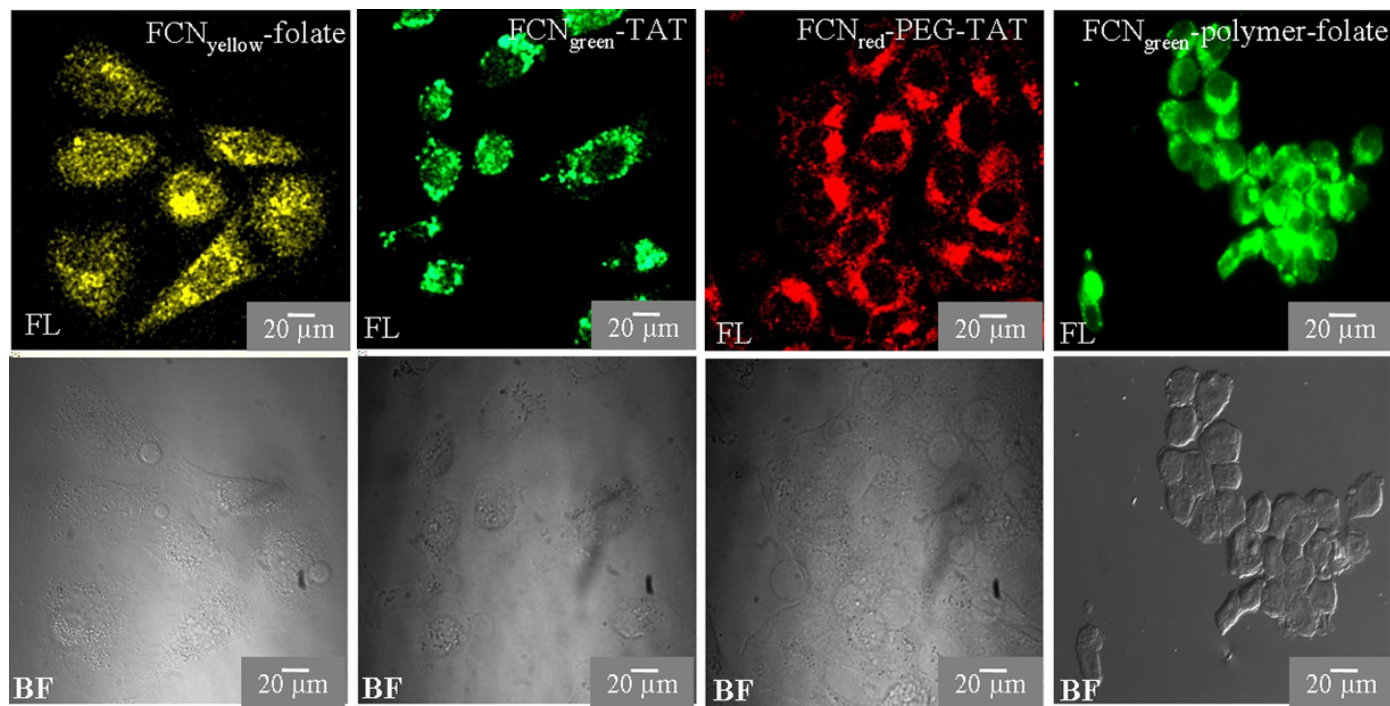

Figure 5 Carbon nanoparticle as fluorescent cell label. FCNs are incubated with HeLa cells for 3-6 hours and labeled cells are imaged under fluorescence microscope. Cells are imaged under bright field (BF) and fluorescence (FL) mode with confocal or Apotome microscope. Details of functional FCNs are described in text and supporting information.

conventional fluorescence microscope. (Figure 5) Control experiments show that the FCNs have very low non-specific binding to cells because of their small hydrodynamic diameter and low surface charge ${ }^{47}$. (Supplementary Figure S20) In addition, TAT functionalisation increases cell labelling and uptake, and folate functionalisation offers selective labelling of cells that have folate receptors ${ }^{47}$. A cytotoxicity study was performed with a conventional methylthiazolyldiphenyl-tetrazolium bromide- (MTT-) based assay that relies on the colour change of MTT by mitochondrial succinate dehydrogenase $e^{48}$. The results show that FCNs are non-toxic at doses substantially higher than the usual concentrations $(<0.2 \mathrm{mg} / \mathrm{mL})$ required for cell labelling studies. (Figure 6 ) These results clearly show that different functionalised FCNs can be synthesised and used as nontoxic labels for cellular and in vivo imaging applications.

\section{Discussion}

We tried to correlate the origin of the tunable emission and the high fluorescence quantum yield with the particle size and chemical composition of the FCNs. Our results show that there is a correlation

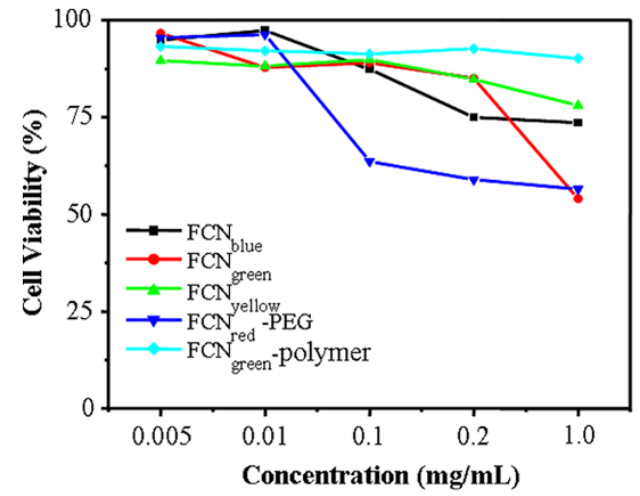

Figure 6 Low cytotoxicity of fluorescent carbon nanoparticle. MTT based cytotoxicity assay for different FCN, showing that they are non-toxic at substantial high dose as compared to usual required concentration $(<0.2 \mathrm{mg} / \mathrm{mL})$ for cell labelling. Some toxicity for $\mathrm{FCN}_{\text {green }}$ and $\mathrm{FCN}_{\text {red }}$-PEG are observed at high dose, possibly due to primary amine groups present on their surface that are known to induce cationic surface and toxicity. between the particle size and the emission colour. As the size of the FCNs increase, the absorption edge, the excitation maxima and the emission maxima are red shifted. The maximum size limit that yields a fluorescent carbon particle is typically $\sim 10 \mathrm{~nm}$; thus, synthetic methods that produce carbon nanoparticles $>10 \mathrm{~nm}$ are either nonfluorescent or are weakly fluorescent. However, size is not the only factor that dictates the emission. The presence of other elements, such as oxygen, nitrogen and phosphorous, and their relative ratios in the carbon matrix dictate the quantum yield. (Table 1) In addition, there exists a correlation between the existence of defect sites and the emission. The presence of defect sites is evident from the EPR signal that corresponds to free electrons and the high ratio of the Raman D to $G$ bands. The high number of $\mathrm{sp}^{3}$ carbons and the presence of oxygen, nitrogen and phosphorous would enhance the defect sites ${ }^{4}$. Based on these observations, a tentative explanation for the tunable emission can be given as follows: As particle size increases, the extent of the $\mathrm{sp}^{2}$ domain in the $\mathrm{sp}^{3}$ matrix increases. This increase in the $\mathrm{sp}^{2}$ domain increases the number of conjugated double bonds. The role of oxygen, nitrogen and phosphorous is to control the $\mathrm{sp}^{2}$ domain by controlling the extent of the $\mathrm{sp}^{3}$ carbon matrix and/or controlling the defect sites ${ }^{4}$. Thus, the key features of the present synthetic method are the restriction of the carbon particle size to $<5-10 \mathrm{~nm}$ and the effective incorporation of other elements that induce defect sites in such a small-sized particle.

The FCN-based probes presented have properties that are far superior to those of other previously reported nanoparticle-based probes. Gold nanoparticle-based probes, which afford dark field imaging, have limited applications for cellular imaging because of the high scattering background from the cell ${ }^{49}$. Semiconductor nanocrystal- (or quantum dot-) based fluorescent probes are limited by the use of toxic cadmium?. Fluorescent nanoprobes based on doped semiconductor nanocrystals ${ }^{50}$, fluorescent gold clusters ${ }^{51}$ and fluorescent silicon nanoparticles ${ }^{52}$ are under development and have yet to exhibit performances similar to that of quantum dots. In contrast, FCN-based probes are composed of nontoxic carbon materials that have tunable fluorescence and have a hydrodynamic diameter $<15 \mathrm{~nm}$. We have convincingly demonstrated that the fluorescence of the FCNs is size-dependent, that the fluorescence is stable to chemical modification and that similar to quantum dots, various functionalised FCNs with tunable emission can be prepared. We have also demonstrated that functionalised FCNs can be used as fluorescent biological labels to image and detect molecules inside 
cells. These labelling applications indicate that FCNs can be powerful nontoxic alternatives to semiconductor nanocrystals. Additionally, the smaller sizes of the FCNs should be more powerful for subcellular targeting and have better renal clearance; these properties are often limited for semiconductor nanocrystals ${ }^{53}$. The synthetic and functionalisation approach presented here produces high-quality FCNs in milligram to gram scale, and production on larger scales is an option. Thus, the present approach would make high-quality FCNs available on a large scale for testing in various biomedical applications.

In conclusion, we have demonstrated that carbon-based nontoxic fluorescent nanoparticles can be a powerful alternative to cadmiumbased toxic semiconductor nanocrystals. Highly fluorescent carbon nanoparticles with tunable visible emission from blue to red have been synthesised in gram scale and transformed into various biological labels. The small size, stable emission and low toxicity of these nanoprobes give these nanoprobes advantages over the widely used semiconductor nanocrystals. Future work should focus on utilising the full potential of these nanomaterials, particularly in the biomedical sciences.

\section{Methods}

FCN synthesis. Hydrophobic $\mathrm{FCN}_{\text {blue }}$ and $\mathrm{FCN}_{\text {green }}$ were synthesised by mixing 50-200 mg of carbohydrate with 1-2 g of octadecylamine and octadecene and then heating the mixture at $70-300^{\circ} \mathrm{C}$ for $10-30$ minutes. For example, $\mathrm{FCN}_{\text {blue }}$ was synthesised by mixing $150 \mathrm{mg}$ of carbohydrate, $0.5 \mathrm{~g}$ of octadecylamine and $10 \mathrm{~mL}$ of octadecene and heating the mixture at $80^{\circ} \mathrm{C}$ for 20 minutes. Similarly, $\mathrm{FCN}_{\text {green }}$ was synthesised by mixing $160 \mathrm{mg}$ of carbohydrate and $1.6 \mathrm{~g}$ of octadecylamine and heating the mixture at $80^{\circ} \mathrm{C}$ for 10 minutes. Next, the reaction mixture was cooled to room temperature, and the dark brown solid was mixed with $5 \mathrm{~mL}$ of chloroform or acetone and was shaken vigorously. The soluble FCNs were extracted using organic solvent, and octadecylamine remained as an insoluble white solid. The organic solvent was then evaporated, and the brown, solid FCNs were solubilised in common organic solvents such as acetone, chloroform, toluene or cyclohexane. However, these hydrophobic FCNs are partially soluble in methanol and ethanol and are insoluble in water. The hydrophobic FCNs were further purified by column chromatography. In this separation process, silica gel (60-120 mesh) was used as the stationary phase, and dichloromethane and methanol were used as the mobile phase. This process separates most of the remaining octadecylamine from the FCNs.

Hydrophilic $\mathrm{FCN}_{\text {blue }}$ and $\mathrm{FCN}_{\text {green }}$ were synthesised by dissolving $0.2-1.6 \mathrm{~g}$ of carbohydrate in 3-10 $\mathrm{mL}$ of water, and the $\mathrm{pH}$ was made acidic or basic using solutions of $\mathrm{NaOH}$ or $\mathrm{HCl}$. Next, the solution was heated at $\sim 70-90^{\circ} \mathrm{C}$ for $20-30$ minutes. Alternatively, 1-4 mL of concentrated carbohydrate solution $(350 \mathrm{mg} / \mathrm{mL})$ was mixed with $5-20 \mathrm{~mL}$ of concentrated sulphuric acid and heated to $70-90^{\circ} \mathrm{C}$ for 20-60 minutes. For example, $\mathrm{FCN}_{\text {blue }}$ was synthesised by dissolving $1.5 \mathrm{~g}$ of carbohydrate in $5 \mathrm{~mL}$ of water, the $\mathrm{pH}$ was made acidic by the addition of $\mathrm{HCl}$, and the solution was heated at $90^{\circ} \mathrm{C}$ for 60 minutes. Similarly, FCN $_{\text {green }}$ was synthesised by dissolving $0.7 \mathrm{~g}$ of carbohydrate in $5 \mathrm{~mL}$ of water, the $\mathrm{pH}$ was made basic by the addition of $\mathrm{NaOH}$ solution, and the solution was heated at $90^{\circ} \mathrm{C}$ for 20 minutes. $\mathrm{FCN}_{\text {green }}$ was also synthesised by mixing 1-4 $\mathrm{mL}$ of concentrated carbohydrate solution $(350 \mathrm{mg} / \mathrm{mL})$ with $5-20 \mathrm{~mL}$ of concentrated sulphuric acid and then heating the solution at $70-90^{\circ} \mathrm{C}$ for $5-20$ minutes. The yellow/brown solution was neutralised by the addition of $\mathrm{NaOH}$ solution and was then dialysed to remove the excess salts and reagents.

Hydrophilic FCN $\mathrm{Fellow}_{\text {and }}$ and $\mathrm{FCN}_{\text {red }}$ were synthesised by mixing 1-4 $\mathrm{mL}$ of aqueous carbohydrate solution $(200-400 \mathrm{mg} / \mathrm{mL})$ with $2.5-10 \mathrm{~mL}$ of concentrated phosphoric acid and heating the solution at $\sim 80-90^{\circ} \mathrm{C}$ for $15-60$ minutes. For example, $700 \mathrm{mg}$ of carbohydrate was dissolved in $2 \mathrm{~mL}$ of water, mixed with $5 \mathrm{~mL}$ of concentrated phosphoric acid and heated at $90^{\circ} \mathrm{C}$ for 15 minutes to obtain $\mathrm{FCN}_{\text {yellow }}$. Similarly, $200 \mathrm{mg}$ of carbohydrate and $0.5 \mathrm{~mL}$ of PEG-diamine were dissolved in $2 \mathrm{~mL}$ of concentrated phosphoric acid and heated at $90^{\circ} \mathrm{C}$ for 6 hours to obtain $\mathrm{FCN}_{\text {red. }}$. The synthesised yellow/brown solution was neutralised with $\mathrm{NaOH}$. The resulting precipitate was repeatedly washed or the resulting solution was dialysed to remove excess salt.

QY measurement. The quantum yields of the samples were measured in reference to quinine sulphate $(\mathrm{QY}=58 \%$ at $354 \mathrm{~nm}$ excitation) and fluorescein $(\mathrm{QY}=95 \%$ at $465 \mathrm{~nm}$ excitation). The formula used for QY measurements is as follows:

$$
(\mathrm{QY})_{\mathrm{Sm}}=(\mathrm{QY})_{\mathrm{St}} \times\left[(\mathrm{PL} \text { area } / \mathrm{OD})_{\mathrm{Sm}} /(\mathrm{PL} \text { area } / \mathrm{OD})_{\mathrm{St}}\right] \times \eta_{\mathrm{sm}}^{2} / \eta_{\mathrm{st}}^{2}
$$

where Sm indicates the sample, St indicates the standard, $\eta$ is the refractive index of the solvent, and PL area and OD are the fluorescence area and absorbance value, respectively. Quinine sulphate was used as a reference for $\mathrm{FCN}_{\mathrm{blue}}, \mathrm{FCN}_{\text {green }}$ and $\mathrm{FCN}_{\text {red }}$, and the fluorescein was used as a reference for $\mathrm{FCN}_{\text {yellow. }}$

FCN functionalisation. Water soluble FCNs with primary amines on their surfaces were used for covalent functionalisation with affinity molecules. Hydrophobic FCNs, which are water-insoluble and do not have this amine functionality, have been transformed into water-soluble and primary amine-terminated FCNs with a polymer coating. In a typical procedure for the synthesis of the polymer coating, $1 \mathrm{~mL}$ of hydrophobic FCNs in chloroform $(\sim 10 \mathrm{mg} / \mathrm{mL})$ was mixed with $0.5 \mathrm{~mL}$ of polymaleicanhydride-1-octadecene solution $(80 \mathrm{mg}$ dissolved in $0.5 \mathrm{~mL}$ chloroform), and the solution was sonicated for 5 minutes. Next, $O, O^{\prime}$-bis (2-amino propyl) polypropylene glycol-block-polyethylene glycol-block-polypropylene glycol (PEG-diamine) (120 mg PEG-diamine dissolved in $0.5 \mathrm{~mL}$ chloroform) was mixed with the FCN solution. The solution was evaporated to dryness overnight, $2-3 \mathrm{~mL}$ of aqueous $\mathrm{Na}_{2} \mathrm{CO}_{3}$ solution was added, and the solution was maintained at room temperature for 1-2 hours. The FCNs were completely dissolved in water, and this aqueous solution was used for functionalisation.

Functionalisation of the primary amine-terminated FCNs was achieved with wellknown bioconjugation chemistry. Folic acid-functionalisation of FCNs was achieved by preparing $\mathrm{N}$-hydroxysuccinimide-folate (NHS-folate) and then reacting NHSfolate with the primary amine-terminated FCNs. Typically, 1-2 $\mathrm{mL}$ of an aqueous solution of FCN was prepared in borate buffer at $\mathrm{pH} 9$ and was mixed with $0.1-$ $0.3 \mathrm{~mL}$ of $1 \mathrm{mM}$ dimethylformamide solution of NHS-folate. After reacting overnight, the solution was dialysed with a dialysis membrane (MWCO 12-14 kDa from Sigma-Aldrich) to remove the excess reagents.

TAT peptide (CHHHHHHHHHHGRKKRRQRRR, MW 2871, 95\% purity) functionalisation was achieved with the well-known 4-maleimido-butyric acid Nhydroxysuccinimide ester reagent. The maleimido group of this reagent reacts with the thiol group of the cysteine-terminated TAT peptide, and the N-hydroxysuccinimide group reacts with the primary amine group of the FCNs. These reactions result in a covalent linkage between the FCNs and the TAT peptide. Typically, 1$2 \mathrm{~mL}$ of aqueous solution of FCNs was mixed with $25-100 \mu \mathrm{L}$ of a dimethylformamide solution of the conjugation reagent $(2-5 \mathrm{mg} / \mathrm{mL})$, and after 30 minutes, 50$100 \mu \mathrm{L}$ of TAT peptide solution was added to the mixture. After reacting overnight, the solution was dialysed with a dialysis membrane (MWCO $12-14 \mathrm{kDa}$ ) to remove the excess reagents and unbound peptide.

Labelling and imaging applications of FCNs. HeLa and other cells were cultured in DMEM medium with $10 \%$ fetal bovine serum and $1 \%$ penicillin streptomycin with $5 \% \mathrm{CO}_{2}$ and at $37^{\circ} \mathrm{C}$. The cells were cultured overnight in a chamber slide with $0.5 \mathrm{~mL}$ of media and then were mixed with $10-100 \mu \mathrm{L}$ of FCN solution and were incubated for 1-6 hours. Next, the cells were washed with buffer solution and fresh culture media was added. The labelled cells were imaged with an Olympus IX 81 or Carl Zeiss Apotome Imager. Z1 microscope or a Nikon AIR Laser Scanning confocal microscope.

Cytotoxicity assay. The cells were treated with different doses of FCN solutions in wells and were incubated for 24 hours. The cells attached to each plate were treated with $50 \mu \mathrm{L}$ of freshly prepared methylthiazolyldiphenyl-tetrazolium bromide (MTT) solution $(5 \mathrm{mg} / \mathrm{mL})$ after incubation and were incubated again for 3 hrs to allow violet- coloured formazan to form. Next, the supernatant was carefully removed, the formazan was dispersed in a 50\% aqueous DMF solution, and the absorbance was measured at $570 \mathrm{~nm}$ with a Microplate reader. The optical density was correlated with the cell viability, under the assumption of $100 \%$ viability for the control sample without any FCNs.

1. Baker, S. N. \& Baker, G. A. Luminescent carbon nanodots: Emergent nanolights. Angew. Chem. Int. Ed. 49, 6726-6744 (2010).

2. Wang, X. et al. Bandgap-like strong fluorescence in functionalized carbon nanoparticles. Angew Chem. Int. Ed. 49, 5310-5314 (2010).

3. Li, Q. et al. Photoluminescent carbon dots as biocompatible nanoprobes for targeting cancer cells in vitro. J. Phys. Chem. C 114, 12062-12068 (2010).

4. Cao, L., Meziani, M. J., Sahu, S. \& Sun, Y.-P. Photoluminescence properties of graphene versus other carbon nanomaterials. Acc. Chem. Res. 46, 171-180 (2013).

5. Krysmann, M. J. et al. Formation mechanism of carbogenic nanoparticles with dual photoluminescence emission. J. Am. Chem. Soc. 134, 747-750 (2012).

6. Yu, B. Y. \& Kwak, S.-Y. Carbon quantum dots embedded with mesoporous hematite nanospheres as efficient visible light-active photocatalysts. J. Mater. Chem. 22, 8345-8353 (2012).

7. Guo, X. et al. Facile access to versatile fluorescent carbon dots toward lightemitting diodes. Chem. Commun. 48, 2692-2694 (2012).

8. Zhu, A. et al. Carbon-dot-based dual-emission nanohybrid produces a ratiometric fluorescent sensor for in vivo imaging of cellular copper ions. Angew. Chem. Int. Ed. 51, 7185-7189 (2012).

9. Medintz, I. L., Uyeda, H. T., Goldman, E. R. \& Mattoussi, H. Quantum dot bioconjugates for imaging, labeling and sensing. Nat. Mater. 4, 435-446 (2005).

10. Pelley, J. L., Daar, A. S. \& Saner, M. A. State of academic knowledge on toxicity and biological fate of quantum dots. Toxicological Sciences 112, 276-296 (2009).

11. Diederich, F. \& Thilgen, C. Covalent fullerene chemistry. Science 271, 317-323 (1996).

12. Tasis, D., Tagmatarchis, N., Bianco, A. \& Prato, M. Chemistry of carbon nanotubes. Chem. Rev. 106, 1105-1136 (2006).

13. Dreyer, D. R., Park, S., Bielawski, C. W. \& Ruoff, R. S. The chemistry of graphene oxide. Chem. Soc. Rev. 39, 228-240 (2010).

14. Gruber, A. et al. Scanning confocal optical microscopy and magnetic resonance on single defect centers. Science 276, 2012-2014 (1997). 
15. Yu, S. J. et al. Bright fluorescent nanodiamonds: No photobleaching and low cytotoxicity. J. Am. Chem. Soc. 127, 17604-17605 (2005).

16. Sun, Y. P. et al. Quantum-sized carbon dots for bright and colorful photoluminescence. J. Am. Chem. Soc. 128, 7756-7757 (2006).

17. Liu, H., Ye, T. \& Mao, C. Fluorescent carbon nanoparticles derived from candle soot. Angew. Chem. Int. Ed. 46, 6473-6475 (2007).

18. Ray, S. C., Saha, A., Jana, N. R. \& Sarkar, R. Fluorescent carbon nanoparticles: Synthesis, characterization, and bioimaging application. J. Phys. Chem. C 113 18546-18551 (2009).

19. Selvi, B. R. et al. Intrinsically fluorescent carbon nanospheres as a nuclear targeting vector: Delivery of membrane-impermeable molecule to modulate gene expression in vivo. Nano Lett. 8, 3182-3188 (2008).

20. Peng, H. \& Travas-Sejdic, J. Simple aqueous solution route to luminescent carbogenic dots from carbohydrates. Chem. Mater. 21, 5563-5565 (2009).

21. Zhang, J. et al. Controlled synthesis of green and blue luminescent carbon nanoparticles with high yields by the carbonization of sucrose. New J. Chem. 34, 591-593 (2010).

22. Yang, Z.-C., Li, X. \& Wang, J. Intrinsically fluorescent nitrogen-containing carbon nanoparticles synthesized by a hydrothermal process. Carbon 49, 5207-5212 (2011).

23. Yang, Y. et al. One-step synthesis of amino-functionalized fluorescent carbon nanoparticles by hydrothermal carbonization of chitosan. Chem. Commun. $\mathbf{4 8}$, 380-382 (2012).

24. Bourlinos, A. B. et al. Photoluminescent carbogenic dots. Chem. Mater. 20 4539-4541 (2008).

25. Bourlinos, A. B. et al. Surface functionalized carbogenic quantum dots. Small 4, 455-458 (2008).

26. Wang, F. et al. One-Step synthesis of highly luminescent carbon dots in noncoordinating solvents. Chem. Mater. 22, 4528-4530 (2010).

27. Pan, D. et al. Observation of $\mathrm{pH}-$-, solvent-, spin-, and excitation-dependent blue photoluminescence from carbon nanoparticles. Chem. Commun. 46, 3681-3683 (2010).

28. Zhang, B., Chun-yan Liu, C.-Y. \& Liu, Y. A novel one-step approach to synthesize fluorescent carbon nanoparticles. Eur. J. Inorg. Chem. 26, 4411-4414 (2010).

29. Liu, R. et al. An aqueous route to multicolor photoluminescent carbon dots using silica spheres as carriers. Angew. Chem. Int. Ed. 48, 4598-4601 (2009).

30. Li, H. et al. One-step ultrasonic synthesis of water-soluble carbon nanoparticles with excellent photoluminescent properties. Carbon 49, 605-609 (2011).

31. Wang, X. et al. Microwave assisted one-step green synthesis of cell-permeable multicolour photoluminescent carbon dots without surface passivation reagents. J. Mater. Chem. 21, 2445-2450 (2011).

32. Chandra, S. et al. Tuning of photoluminescence on different surface functionalized carbon quantum dots. RSC Advances 2, 3602-3606 (2012).

33. Fang, Y. et al. Easy synthesis and imaging applications of cross-linked green fluorescent hollow carbon nanoparticles. ACS Nano 6, 400-409 (2012).

34. Li, H. et al. Water-soluble fluorescent carbon quantum dots and photocatalyst design. Angew. Chem. Int. Ed. 49, 4430-4434 (2010).

35. Bao, L. et al. Electrochemical tuning of luminescent carbon nanodots: from preparation to luminescence mechanism. Adv. Mater. 23, 5801-5806 (2011).

36. Peng, J. et al. Graphene quantum dots derived from carbon fibers. Nano Lett. 12 844-849 (2012).

37. Zhuo, S., Shao, M. \& Lee, S.-T. Upconversion and downconversion fluorescent graphene quantum dots: Ultrasonic preparation and photocatalysis. ACS Nano 6 , 1059-1064 (2012).

38. Hens, S. C., Lawrence, W. G., Kumbhar, A. S. \& Shenderova, O. Photoluminescent nanostructures from graphite oxidation. J. Phys. Chem. C, 116, 20015-20022 (2012).

39. Sun, X. \& Li, Y. Colloidal carbon spheres and their core/shell structures with noble metal nanoparticles. Angew. Chem. Int. Ed. 43, 597-601 (2004).

40. Jana, N. R., Chen, Y. F. \& Peng, X. G. Size- and shape-controlled magnetic $(\mathrm{Cr}, \mathrm{Mn}, \mathrm{Fe}, \mathrm{Co}, \mathrm{Ni})$ oxide nanocrystals via a simple and general approach. Chem Mater. 16, 3931-3935 (2004).
41. Lakowicz, J. R. Principles of fluorescence spectroscopy. Kluwer Academic/Plenum Publishers, New York, 2nd Ed. (1999).

42. Yu, W. W., Qu, L., Guo, W. \& Peng. X. Experimental determination of the extinction coefficient of CdTe, CdSe, and CdS nanocrystals. Chem Mater. 15, 2854-2860 (2003).

43. Jana, N. R., Gearheart, L. \& Murphy, C. J. Seeding growth for size control of 5-40 nm diameter gold nanoparticles. Langmuir 17, 6782 (2001).

44. Pellegrino, T. et al. Hydrophobic nanocrystals coated with an amphiphilic polymer shell: A general route to water soluble nanocrystals. Nano Lett. 4, 703-707 (2004).

45. Bhunia, S. K. \& Jana, N. R. Peptide-functionalized colloidal graphene via interdigited bilayer coating and fluorescence turn-on detection of enzyme. ACS Appl. Mater. Interfaces 3, 3335-3341 (2011).

46. Selvan, S. T., Tan, T. T. Y., Yi, D. K. \& Jana, N. R. Functional and multifunctional nanoparticles for bioimaging and biosensing. Langmuir 26, 11631-11641 (2010).

47. Jana, N. R. Design and development of quantum dots and other nanoparticles based cellular imaging probe. Phys. Chem. Chem. Phys. 13, 385-396 (2011).

48. Sladowski, D., Steer, S. J., Clothier, R. H. \& Balls, M. An improved MTT assay. J. Immunol. Methods 157, 203-207 (1993).

49. Alkilani, A. M., Lohse, S. E. \& Murphy, C. J. The gold standard: Gold nanoparticle libraries to understand the nano-bio interface. Acc. Chem. Res. ASAP (2012) DOI: $10.1021 / a r 300015 b$

50. Srivastava, B. B. et al. Highly luminescent Mn-doped ZnS nanocrystals: gramscale synthesis. J. Phys. Chem. Lett. 1, 1454-1458 (2010).

51. Palmal, S. et al. Thiol-directed synthesis of highly fluorescent gold clusters and their conversion into stable imaging nanoprobes. Chem. Eur. J. 19, 943-949 (2013).

52. Erogbogbo, F. et al. In Vivo targeted cancer imaging, sentinel lymph node mapping and multi-channel imaging with biocompatible silicon nanocrystals. ACS Nano 5, 413-423 (2011).

53. Choi, H. S. et al. Design considerations for tumour-targeted nanoparticles. Nat Nanotechnol. 5, 42-47 (2010).

\section{Acknowledgements}

This work is financially supported by DST and DBT, Government of India. SKB and AS thank CSIR, India and ARM thanks DST and IACS for their research fellowships. Authors would like to thank Dr. Nihar R. Jana of National Brain Research Centre, Manesar, India for providing assistance in cellular imaging. Dr Samir Adhya and Anupam Chatterjee of IICB-Kolkata are acknowledged for providing confocal microscopic study.

\section{Author contributions}

N.R.J. designed the experiments with the initial discussion with S.C.R.; S.K.B. and A.S. performed the FCN synthesis experiments and A.R.M. performed the labeling/imaging experiments. N.R.J. analyzed the data with the help of S.C.R. and prepared the manuscript All authors discussed the results.

\section{Additional information}

Supplementary information accompanies this paper at http://www.nature.com/ scientificreports

Competing financial interests: The authors declare no competing financial interests. License: This work is licensed under a Creative Commons Attribution-NonCommercial-NoDerivs 3.0 Unported License. To view a copy of this license, visit http://creativecommons.org/licenses/by-nc-nd/3.0/

How to cite this article: Bhunia, S.K., Saha, A., Maity, A.R., Ray, S.C. \& Jana, N.R. Carbon Nanoparticle-based Fluorescent Bioimaging Probes. Sci. Rep. 3, 1473; DOI:10.1038/ srep01473 (2013). 